\title{
KINEMATIC AND SINGULARITY ANALYSIS OF PROM-120 - A PARALLEL ROBOTIC MANIPULATOR WITH 2-PRU/PRS KINEMATIC CHAINS
}

\author{
Adriyan $^{1}$, and Sufiyanto ${ }^{2}$ \\ 1,2 Department of Mechanical Engineering, Sekolah Tinggi Teknologi Nasional Jambi \\ Jl. Kapt. Patimura No. 100, Rawasari, Kota Jambi, Indonesia, 36125 \\ E-mail: adriyan0686@gmail.com
}

\begin{abstract}
$P R o M-120$ was a parallel robotic manipulator which constructed by 2-PRU/PRS kinematic chains. The mobility of this manipulator was 3 DOF, i.e. 1 translational DOF, and 2 rotational DOF (1T2R). The objective of this research was to analyze kinematic, and singularity of PRoM-120 based on its geometrical configuration. Inverse kinematics and velocity analysis were carried out by applying loop vector equation. Meanwhile, all screw systems existed in PRoM-120 were derived using screw theory. Singularity analysis revealed that PRoM-120 had no constraint singularity within its workspace. The kinematic singularity of PRoM-120 showed similar condition to transmission singularity. Three types of both singularities were examined and presented for general condition and selected numerical value of kinematic constants having by PRoM-120. The selected numerical values of those kinematic constants showed only one pose for the singular condition, which was the direct kinematic or the output transmission singularity.
\end{abstract}

Keywords: PRoM-120, Screw System, Kinematic Analysis, Singularity Analysis.

INTRODUCTION

A parallel robotic manipulator, also known parallel manipulator, or parallel mechanism (PM in short), is a type of robotic manipulator which constructed by two or more kinematic chains that connect a fixed base to a platform which forms a closed kinematic chain. This type of manipulator offers several advantages over its counterpart, which is a serial robotic manipulator. These advantages are in term of high payload to weight ratio, better stiffness, better precision, and high acceleration, while a smaller working space is a major drawback [1].

The first parallel manipulator is a 6 degree of freedom (DOF) which had 3 translational motion and 3 rotational motion in the space. PM with the mobility of less than 6 , or known as lower DOF PM, becomes more popular more than last three decades [2]-[7]. It was due to the application of PM which required for manipulation of an object in space less than 6 DOF. Using lower DOF PM means that fewer actuators required to be installed on the manipulator base.

Three DOF object manipulation in space can be one of pure translational motion (3T), pure rotational motion $(3 \mathrm{R})$, or combined motion, i.e. 1 translational and 2 rotational motion (1T2R) or 2 translational and 1 rotational motion (2T1R). The 3 DOF PM with combined motion has inherent kinematic features which called as parasitic motion and firstly coined by Carreterro et al. [3]. Afterward, Li et al. [4] presented the number and direction of parasitic motion in the 3 DOF PM in relation to different limb arrangements or limb planes. There were seven kinds of PMs or subcategories of limb planes that later be classified into four categories based on the geometrical condition of the PMs. An undesired motion produced by the parasitic motion can reduce manipulation accuracy, quality, and difficulties in calibration [4]. Real-time compensation can be used to counter the problem caused by this parasitic motion.

Singularity analysis was applied to examine the kinematic performance of the PMs. The PMs at singular conditions must be avoided because the kinematic performance of the PMs will degenerate. There are three types of singularity used to evaluate the kinematic performance of the PMs. These three types are a Jacobian-based (kinematic) singularity, a constraint singularity, and a transmission singularity.

The kinematic singularity occurs mathematically when the rank of a Jacobian matrix is deficient [8]. Then, the constraint singularity can be found if the wrench systems 
of the PMs become linearly dependent [9]. At accomplish such an application, a PRoM-120 last, transmission singularity which proposed by was proposed. PRoM-120 is a parallel robotic Liu et al. [10] is applied to evaluate the ability of manipulator which constructed by 2-PRU/PRS the PMs to transmit motion and force from kinematic chains. This manipulator is a 3 DOF actuated joints to the platform. Then, Xu et al. PM with combined motions, i.e. 1 translational [5] revealed a close relationship between DOF and 2 rotational DOF.

transmission singularity and kinematic singularity of the PMs. It was revealed that the inverse, the direct, and the combined kinematic singularity were akin to the input, the output, and the combined transmission singularity, respectively.

An analytical approach to PRoM-120 was presented in this paper to obtain its kinematic features and its singularity characteristics. A loop vector equation was applied to determine the inverse kinematic solution and velocity of PRoM-120. Then, screw theory was used to

The application of PMs with combined obtain screw systems of PRoM-120. At last, motion, especially 1T2R PMs, has found singularity analysis of PRoM-120 was several areas such as machine tools [5], presented to describe its kinematic telescope [3], and vibration testing [6], [7]. This performance. It was applied to general and paper attempted to apply the PMs in a medical selected value of kinematic constants owned by application such as for bone cutting. To PRoM-120.
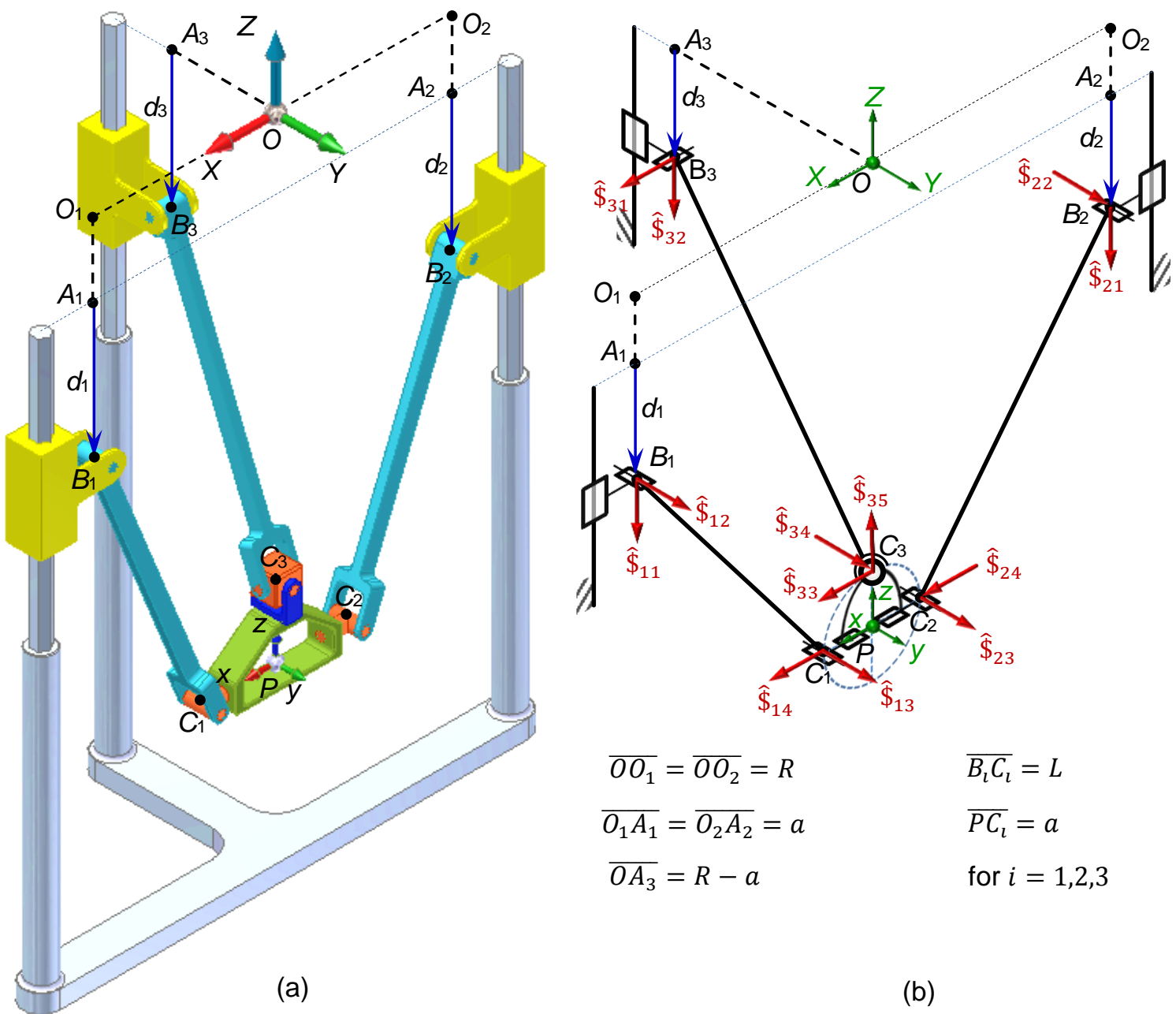

Figure 1. The geometrical architecture of PRoM-120 with 2-PRU/PRS kinematic chain: (a) simplified CAD model and (b) its kinematic diagram with the unit screw on each joint. 


\section{METHODS}

\section{Configuration of PRoM-120}

The kinematic architecture of PRoM-120 was shown in Figure 1. This manipulator was constructed by three limbs, one fixed base, and one platform. The first two limbs shared the same kinematic chain which was PRU chain and lied on the same plane, $X Z$-plane. The third limb was composed by PRS kinematic chain and set on the $Y Z$-plane.

To describe the kinematic properties of PRoM-120, a fixed reference frame, $O-X Y Z$, was assigned to the base. A moving reference frame, $P-x y z$, was set on the platform as depicted in Figure 1 (b). All prismatic joints located on the fixed base were active joints. The U-joint on the first two limbs could be decomposed into two R-joints that the joint axis intersected with each other. These last R-joints on both limbs had the same axis of the moving frame, which was $x$-axis. The S-joint on the third limb could be represented as three $R$-joints that intersect with and perpendicular to each other.

\section{Kinematic and Singularity Analysis}

The inverse kinematic problem (IKP) of PRoM-120 was obtained by setting the loop vector equation for each limb, that had described in Figure 1. Solving for actuated joint spaces, $\left(d_{1} ; d_{2} ; d_{3}\right)$, gave the inverse kinematic solution of PRoM-120. Meanwhile, the velocity analysis of the manipulator can be obtained by taking the derivative of the inverse kinematic solution with respect to time once.

A screw is a dual vector which constructed by a unit vector along the direction of screw axis, $\mathbf{s}$, and a moment of the unit vector $\mathbf{s}$ about a point of reference, $\mathbf{m}$ [11]. It is stated mathematically as

$\$=\rho(\mathbf{s} ; \quad \mathbf{m})=\rho(\mathbf{s} ; \quad \mathbf{r} \times \mathbf{s}+h \mathbf{s})$,

with $\mathbf{r}$ is the position vector between the unit vector $\mathbf{s}$ towards the point of reference, $h$ denotes the pitch of the screw $\$$, and $\rho$ defines the magnitude of the screw. For a unit screw, $\rho$ is equal to 1 , and symbolized by $\widehat{\$}$. A reciprocal screw product is a product of the screw $\$$ and another $i^{\text {th }}$ screw $\$_{i}^{r}$ which satisfies the following relation

$\$ \circ \$_{i}^{r}=\rho \rho_{i}^{r}\left(\mathbf{s} \cdot \mathbf{m}_{i}^{r}+\mathbf{s}_{\boldsymbol{i}}^{r} \cdot \mathbf{m}\right)=0$, with o represents the reciprocal product of two screws. An interested reader can consult [11] for detail description of the screw theory.

Screw system of PRoM-120 was obtained by applying screw theory. It comprised of a limb twist system (LTS), a limb wrench system (LWS), a platform wrench system (PWS), a platform twist system (PTS), an input twist system (ITS), a transmission wrench system (TWS) and an output twist system (OTS).

The Jacobian-based singularity or kinematic singularity was computed by using a Jacobian matrix which derived from the velocity equation. Meanwhile, motion and force transmission-based singularity was calculated from the last three screw systems presented previously. Also, constraint singularity can be analyzed directly from the PWS.

Finally, a selected case of PRoM-120 was simulated by using its kinematic constants as listed in Table 1. These constants referred to the geometrical architecture of PRoM-120 in Figure 1 and were not the optimized values. Then, the travel distance of each prismatic actuator was set from $0 \mathrm{~mm}$ to $200 \mathrm{~mm}$.

Table 1. Kinematic constants of PRoM-120.

\begin{tabular}{rlr} 
No & \multicolumn{1}{c}{ Parameter } & Value \\
1 & Radius of the base $(R)$ & $200 \mathrm{~mm}$ \\
2 & Length of the link $(L)$ & $400 \mathrm{~mm}$ \\
3 & Radius of the platform $(a)$ & $80 \mathrm{~mm}$
\end{tabular}

\section{RESULTS AND DISCUSSIONS}

Inverse Kinematic of PRoM-120

PRoM-120 had the orientation capability in two continuous rotations about $x$-axis and $y$ axis that assigned by rotation angles $\vartheta$ and $\phi$, respectively. The position of point $C_{i}$ in the fixed frame can be written as

$\overrightarrow{O C_{l}}=\overrightarrow{O P}+\mathbf{R}(\vartheta, \phi) \cdot \overrightarrow{P C_{l}}$,

for $i=1 ; 2 ; 3$, with $\mathbf{R}(\vartheta, \phi)$ denoted the rotation matrix between the moving frame and the fixed frame, which defined as

$\mathbf{R}(\vartheta, \phi)=\mathbf{R}(y, \phi) \mathbf{R}(x, \vartheta)=\left[\begin{array}{ccc}c_{\phi} & s_{\phi} s_{\vartheta} & c_{\vartheta} s_{\phi} \\ 0 & c_{\vartheta} & -s_{\vartheta} \\ -s_{\phi} & c_{\phi} s_{\vartheta} & c_{\phi} c_{\vartheta}\end{array}\right]$.

Then, $c_{\vartheta}, s_{\vartheta}, c_{\phi}$, and $s_{\phi}$ were a short notation for cosine and sine of the rotation angles $(\vartheta$ and $\phi)$, 
respectively. Meanwhile, the position of point $P$ in the fixed frame was $\overrightarrow{O P}=\left(\begin{array}{lll}x & y & -z\end{array}\right)^{T}$.

The parasitic motion of PRoM-120 can be found by applying the dot product of eq. (3) with a unit vector, $\widehat{\mathbf{u}}_{i}(i=1 ; 2 ; 3)$. This unit vector was perpendicular to each limb plane of PRoM-120, i.e. $\quad \widehat{\mathbf{u}}_{1}=\widehat{\mathbf{u}}_{2}=\left(\begin{array}{lll}0 & 1 & 0\end{array}\right)^{T}, \quad$ and $\widehat{\mathbf{u}}_{3}=\left(\begin{array}{lll}1 & 0 & 0\end{array}\right)^{T}$ and satisfied the condition $\overrightarrow{O C_{l}} \cdot \widehat{\mathbf{u}}_{i}=0$. Thus, the condition for the parasitic motion of PRoM-120 was given by

$\left\{\begin{array}{l}x=-a c_{\vartheta} s_{\phi} \\ y=0\end{array}\right.$

It showed that the parasitic motion only appeared in the $X$-direction and was regarded as subcategory 2.2 according to [4].

Referring to the geometric description of PRoM-120 as depicted in Figure 1, it can be established the loop vector equation for each limb as given by

$\overrightarrow{B_{l} C_{l}}=-\overrightarrow{O B_{l}}+\overrightarrow{O C_{l}}$,

for $i=1 ; 2 ; 3$, in which $\overrightarrow{B_{l} C_{l}}, \overrightarrow{O B_{l}}\left(=\overrightarrow{O A_{l}}+\overrightarrow{A_{l} B_{l}}\right)$, and $\overrightarrow{O C_{l}}$ denoted the position vector from point $B_{i}$ to $C_{i}$, point $O$ to $B_{i}$, and point $O$ to $C_{i}$, respectively. Applying the dot product to eq. (5) produced

$\overrightarrow{B_{l} C_{l}} \cdot \overrightarrow{B_{l} C_{l}}=\left(-\overrightarrow{O B_{l}}+\overrightarrow{O C_{l}}\right) \cdot\left(-\overrightarrow{O B_{l}}+\overrightarrow{O C_{l}}\right)$,

or in the expanded form for each limb as

$L^{2}=k_{i}^{2}+Z_{i}^{2}$,

for $i=1 ; 2 ; 3$, with $k_{1}=-\left[R-a\left(c_{\phi}-c_{\vartheta} s_{\phi}\right)\right]$, $k_{2}=R-a\left(c_{\phi}+c_{\vartheta} s_{\phi}\right), \quad k_{3}=R-a\left(1+s_{\vartheta}\right)$, $Z_{1}=d_{1}-z+a\left(1-s_{\phi}\right), \quad Z_{2}=d_{2}-z+a(1+$ $\left.s_{\phi}\right)$, and $Z_{3}=d_{3}-z+a c_{\phi} c_{\vartheta}$.

An inverse kinematic solution of the manipulator can be obtained by solving the actuated joint spaces $\left(d_{1} ; d_{2} ; d_{3}\right)$ from eq. (7) for the given platform space $(z ; \vartheta ; \phi)$. It yielded

$\left\{\begin{array}{l}d_{1}=z-a\left(1-s_{\phi}\right)+\lambda_{1} \\ d_{2}=z-a\left(1+s_{\phi}\right)+\lambda_{2} \\ d_{3}=z-a c_{\vartheta} c_{\phi}+\lambda_{3}\end{array}\right.$

with $\lambda_{i}=-\sqrt{L^{2}-k_{i}^{2}}$, for $i=1 ; 2 ; 3$.

\section{Velocity of PRoM-120}

Velocity equation of the manipulator can be found in a simple way by taking the derivative of eq. (8) with respect to time once. Thus, it can be written mathematically as

$$
\left\{\begin{array}{l}
\dot{d}_{1}=\dot{z}+\frac{\partial \lambda_{1}}{\partial \vartheta} \dot{\vartheta}+\left(a c_{\phi}+\frac{\partial \lambda_{1}}{\partial \phi}\right) \dot{\phi} \\
\dot{d}_{2}=\dot{z}+\frac{\partial \lambda_{2}}{\partial \vartheta} \dot{\vartheta}+\left(-a c_{\phi}+\frac{\partial \lambda_{2}}{\partial \phi}\right) \dot{\phi} \\
\dot{d}_{3}=\dot{z}+\left(a s_{\vartheta} c_{\phi}+\frac{\partial \lambda_{3}}{\partial \vartheta}\right) \dot{\vartheta}+a c_{\vartheta} s_{\phi} \dot{\phi}
\end{array},\right.
$$

with $\frac{\partial \lambda_{1}}{\partial \vartheta}=\frac{a k_{1} s_{\vartheta} s_{\phi}}{\lambda_{1}}=\frac{h_{11}}{\lambda_{1}}, \frac{\partial \lambda_{1}}{\partial \phi}=-\frac{a k_{1}\left(s_{\phi}+c_{\vartheta} c_{\phi}\right)}{\lambda_{1}}=$ $\frac{h_{12}}{\lambda_{1}}, \frac{\partial \lambda_{2}}{\partial \vartheta}=\frac{a k_{2} s_{\vartheta} s_{\phi}}{\lambda_{2}}=\frac{h_{21}}{\lambda_{2}}, \frac{\partial \lambda_{2}}{\partial \phi}=\frac{a k_{2}\left(s_{\phi}-c_{\vartheta} c_{\phi}\right)}{\lambda_{2}}=\frac{h_{22}}{\lambda_{2}}$, and $\frac{\partial \lambda_{3}}{\partial \vartheta}=-\frac{a k_{3} c_{\vartheta}}{\lambda_{3}}=\frac{h_{31}}{\lambda_{3}}$.

By rearranging eq. (9) and presenting them in the matrix form, it gave a simple velocity equation which described as

$\mathbf{J}_{q} \dot{\mathbf{q}}=\mathbf{J}_{x} \dot{\mathbf{x}}$

or

$\dot{\mathbf{q}}=\mathbf{J}_{q}^{-1} \mathbf{J}_{x} \dot{\mathbf{x}}=\mathbf{J} \dot{\mathbf{x}}$

with $\mathbf{J}_{q}$ denoted the Jacobian of the inverse kinematic,

$\mathbf{J}_{q}=\left[\begin{array}{ccc}\lambda_{1} & 0 & 0 \\ 0 & \lambda_{2} & 0 \\ 0 & 0 & \lambda_{3}\end{array}\right]$

and $\mathbf{J}_{x}$ was the Jacobian of the direct kinematic,

$$
\mathbf{J}_{x}=\left[\begin{array}{ccc}
\lambda_{1} & h_{11} & h_{12}+a c_{\phi} \lambda_{1} \\
\lambda_{2} & h_{21} & h_{22}-a c_{\phi} \lambda_{2} \\
\lambda_{3} & h_{31}+a s_{\vartheta} c_{\phi} \lambda_{3} & a c_{\vartheta} s_{\phi} \lambda_{3}
\end{array}\right],
$$

Meanwhile, Jacobian of the manipulator, $\mathbf{J}$, could be computed by $\mathbf{J}_{q}^{-1} \mathbf{J}_{x}$, if $\mathbf{J}_{q}$ was not a singular matrix. Then, $\dot{\mathbf{q}}=\left(\begin{array}{lll}\dot{d}_{1} & \dot{d}_{2} & \dot{d}_{3}\end{array}\right)^{T}$ and $\dot{\mathbf{x}}=\left(\begin{array}{lll}\dot{z} & \dot{\vartheta} & \dot{\phi}\end{array}\right)^{T}$ were the vector of actuator velocities and platform velocity, respectively.

\section{Screw System of PRoM-120}

All screw systems were presented with respect to the fixed frame $O-X Y Z$. The screw system which could be constructed by referring to the unit screws as shown in Figure 1 (b) was 
a twist system of each limb or the LTS. It represented the motion screw imposed by each joint in every limb. The LTS for the first two limbs was

$$
\left\{\begin{array}{l}
\widehat{\$}_{i 1}=\left(\begin{array}{llllll}
0 & 0 & 0 ; & 0 & 0 & -1
\end{array}\right) \\
\widehat{\$}_{i 2}=\left(\begin{array}{llllll}
0 & 1 & 0 ; & a+d_{i} & 0 & R
\end{array}\right) \\
\widehat{\$}_{i 3}=\left(\begin{array}{llllll}
0 & 0 & 0 ; & p_{i 3} & 0 & r_{i 3}
\end{array}\right) \\
\widehat{\$}_{i 4}=\left(\begin{array}{llllll}
c_{\phi} & 0 & -s_{\phi} ; & 0 & q_{14} & 0
\end{array}\right)
\end{array},\right.
$$

for $i=1 ; 2$, with $p_{13}=a s_{\phi}+z, p_{23}=-a s_{\phi}+z$, $r_{13}=a\left(c_{\phi}-c_{\vartheta} s_{\phi}\right), \quad r_{23}=-a\left(c_{\phi}+c_{\vartheta} s_{\phi}\right)$, and $q_{14}=-a c_{\vartheta} s_{\phi}^{2}-c_{\phi} z$. Then, the LTS of the third limb was

$$
\left\{\begin{array}{l}
\widehat{\$}_{31}=\left(\begin{array}{llllll}
0 & 0 & 0 ; & 0 & 0 & -1
\end{array}\right) \\
\hat{\$}_{32}=\left(\begin{array}{llllll}
1 & 0 & 0 ; & 0 & -d_{3} & R-a
\end{array}\right) \\
\hat{\$}_{33}=\left(\begin{array}{llllll}
1 & 0 & 0 ; & 0 & q_{33} & r_{33}
\end{array}\right) \\
\hat{\$}_{34}=\left(\begin{array}{llllll}
0 & c_{\vartheta} & s_{\vartheta} ; & p_{34} & 0 & 0
\end{array}\right) \\
\hat{\$}_{35}=\left(\begin{array}{llllll}
e_{35} & -s_{\vartheta} & g_{35} ; & p_{35} & q_{35} & r_{35}
\end{array}\right)
\end{array},\right.
$$

with $q_{33}=a c_{\vartheta} c_{\phi}+z, r_{33}=a s_{\vartheta}, \quad p_{34}=-a s_{\vartheta}^{2}-$ $c_{\vartheta}\left(a c_{\vartheta} c_{\phi}-z\right), \quad e_{35}=c_{\vartheta} s_{\phi}, \quad g_{35}=c_{\vartheta} c_{\phi}$, $p_{35}=-s_{\vartheta} z, \quad q_{35}=c_{\vartheta} s_{\phi}\left(a c_{\vartheta} c_{\phi}-z\right)$, and $r_{35}=a c_{\vartheta} s_{\vartheta} s_{\phi}$.

Applying reciprocal screw product for each LTS which had obtained previously would produce the LWS. It represented the constraint on each limb. The LWS of the first and the second limb was obtained as

$\left\{\begin{array}{l}\hat{\$}_{i 1}^{r}=\left(\begin{array}{llllll}0 & 0 & 0 ; & s_{\phi} & 0 & c_{\phi}\end{array}\right) \\ \hat{\$}_{i 2}^{r}=\left(\begin{array}{llllll}0 & c_{\phi} & 0 ; & p_{W 2} & 0 & 0\end{array}\right)\end{array}\right.$,

for $i=1 ; 2$, and $p_{W 2}=a c_{\vartheta} s_{\phi}^{2}+c_{\phi} z$. It stated that each limb was constrained by one constraint moment and one constraint force. Meanwhile, the LWS of the third limb was given as

$\widehat{\$}_{31}^{r}=\left(\begin{array}{llllll}1 & 0 & 0 ; & 0 & q_{W 3} & r_{33}\end{array}\right)$,

with $q_{W 3}=a c_{\vartheta} c_{\phi}-z$. The reciprocal screw given by eq. (14) indicated that the third limb was restrained by one constraint force.

Hence, the PWS can be formed by taking the union of three LWS in eq. (13) and (14) and finding the linearly independent screw. It gave
$\left\{\begin{array}{l}\hat{\$}_{W 1}=\left(\begin{array}{llllll}0 & 0 & 0 ; & s_{\phi} & 0 & c_{\phi}\end{array}\right) \\ \widehat{\$}_{W 2}=\left(\begin{array}{llllll}0 & c_{\phi} & 0 ; & p_{W 2} & 0 & 0\end{array}\right) \\ \widehat{\$}_{W 3}=\left(\begin{array}{llllll}1 & 0 & 0 ; & 0 & q_{W 3} & r_{33}\end{array}\right)\end{array}\right.$

These three wrenches in eq. (15) showed one constraint moment, $\widehat{\$}_{W 1}$, and two constraint forces, $\widehat{\$}_{W 2}$ and $\widehat{\$}_{W 3}$, that restricted motion of the platform.

The PTS of PRoM-120 can be obtained by applying reciprocal screw product to the PWS which gave

$\left\{\begin{array}{l}\hat{\$}_{M 1}=\left(\begin{array}{llllll}0 & 0 & 0 ; & 0 & 0 & 1\end{array}\right) \\ \widehat{\$}_{M 2}=\left(\begin{array}{llllll}0 & 1 & 0 ; & -q_{W 3} & 0 & 0\end{array}\right) \\ \widehat{\$}_{M 3}=\left(\begin{array}{llllll}-c_{\phi} & 0 & s_{\phi} ; & -r_{33} s_{\phi} & p_{W 2} & 0\end{array}\right)\end{array}\right.$.

Eq. (16) described the motion of PRoM-120 or mobility owned by the manipulator. $\widehat{\$}_{M 1}, \widehat{\$}_{M 2}$, and $\hat{\$}_{M 3}$ defined the number of mobility of PRoM-120 which was 3 DOF and the type of motion i.e. 1 translational motion along $Z$ direction, and 2 rotational motion about $Y$-axis and $X$-axis, respectively.

The ITS can be obtained easily from all actuated joints. PRoM-120 was actuated by linear actuators which drove all prismatic joints. Thus, the ITS for each limb was given by

$\widehat{\$}_{I i}=\widehat{\$}_{i 1}=\left(\begin{array}{llllll}0 & 0 & 0 ; & 0 & 0 & -1\end{array}\right)$,

for $i=1 ; 2 ; 3$.

The TWS of each limb can be determined by finding a screw which has to be linearly independent of each passive LTS and the PWS [10]. The passive LTS of the certain limb can be formed from the LTS itself by excluding the ITS of that limb. Taking reciprocal screw product to the passive LTS resulted in reciprocal screws that must not belong to the PWS, eq. (15). There was a unique screw called as the TWS of each limb

$\left\{\begin{array}{l}\widehat{\$}_{T 1}=\left(\begin{array}{llllll}-k_{1} & 0 & -\lambda_{1} ; & 0 & q_{T 1} & 0\end{array}\right) \\ \widehat{\$}_{T 2}=\left(\begin{array}{llllll}k_{2} & 0 & \lambda_{2} ; & 0 & -q_{T 2} & 0\end{array}\right) \\ \hat{\Phi}_{T 3}=\left(\begin{array}{llllll}0 & k_{3} & \lambda_{3} ; & -p_{T 3} & 0 & 0\end{array}\right)\end{array}\right.$

with $q_{T 1}=p_{13} k_{1}+r_{13} \lambda_{1}, \quad q_{T 2}=p_{23} k_{2}+r_{23} \lambda_{2}$, and $p_{T 3}=q_{W 3} k_{3}+r_{33} \lambda_{3}$. Hence, the TWS of each limb was the transmission force which directed along the link $B_{i} C_{i}(i=1 ; 2 ; 3)$. 
Finally, the OTS of the certain limb can be found by actuating that limb and locking the others. This condition will produce a new wrench system in the manipulator. As an example, the new wrench system for the first limb can be found by employing the PWS, $\left[\hat{\$}_{W 1}, \hat{\$}_{W 2}, \hat{\$}_{W 3}\right]$, and the TWS other than that limb, i.e. $\left[\hat{\$}_{T 2}, \widehat{\$}_{T 3}\right]$. Also, it can be applied for the other two limbs as well. Thus, the new wrench system, $\mathbf{W}_{i}(i=1 ; 2 ; 3)$ can be written as

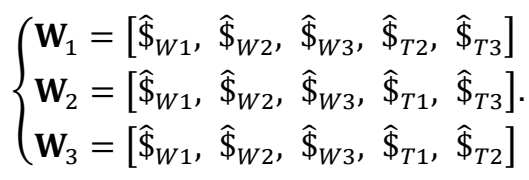

The OTS of the first, the second and the third limb were obtained by applying reciprocal screw product, eq. (2), to $\mathbf{W}_{i}(i=1 ; 2 ; 3)$, respectively. It gave

$\widehat{\$}_{O i}=\left(\begin{array}{llllll}e_{O i} & f_{O i} & g_{O i} ; & p_{O i} & q_{O i} & r_{O i}\end{array}\right)$,

with $e_{O 1}=-c_{\phi} \lambda_{3} \mu_{1}, f_{O 1}=c_{\phi} s_{\vartheta} \lambda_{2} \lambda_{3}+c_{\vartheta} \lambda_{2} k_{3}+$ $s_{\phi} s_{\vartheta} k_{2} \lambda_{3}, \quad g_{O 1}=s_{\phi} \lambda_{3} \mu_{1}, \quad p_{O 1}=-a s_{\phi} s_{\vartheta} \lambda_{3} \mu_{1}-$ $q_{W 3} f_{O 1}, \quad q_{O 1}=p_{W 2} \lambda_{3} \mu_{1}, \quad r_{O 1}=-a\left(c_{\phi} s_{\vartheta} \lambda_{3}+\right.$ $\left.c_{\vartheta} k_{3}\right) \mu_{1}, \quad \mu_{1}=\left(s_{\phi}-c_{\phi} c_{\vartheta}\right) k_{2}+\left(c_{\phi}+c_{\vartheta} s_{\phi}\right) \lambda_{2}$, $e_{O 2}=-c_{\phi} \lambda_{3} \mu_{2}, \quad f_{O 2}=-s_{\phi} s_{\vartheta} k_{1} \lambda_{3}-\left(c_{\vartheta} k_{3}+\right.$ $\left.c_{\phi} s_{\vartheta} \lambda_{3}\right) \lambda_{1}, g_{O 2}=s_{\phi} \lambda_{3} \mu_{2}, \quad p_{O 2}=-a s_{\phi} s_{\vartheta} \lambda_{3} \mu_{2}-$ $q_{W 3} f_{O 2}, \quad q_{O 2}=p_{W 2} \lambda_{3} \mu_{2}, \quad r_{O 2}=-a\left(c_{\phi} s_{\vartheta} \lambda_{3}+\right.$ $\left.c_{\vartheta} k_{3}\right) \mu_{2}, \quad \mu_{2}=\left(s_{\phi}+c_{\phi} c_{\vartheta}\right) k_{1}+\left(c_{\phi}-c_{\vartheta} s_{\phi}\right) \lambda_{1}$, $e_{O 3}=-c_{\phi} \mu_{3}, \quad f_{O 3}=s_{\vartheta} s_{\phi}\left(-k_{1} \lambda_{2}+\lambda_{1} k_{2}\right)$, $g_{03}=s_{\phi} \mu_{3}, \quad p_{03}=-s_{\vartheta} s_{\phi}\left[2 a c_{\phi} \lambda_{1} \lambda_{2}+\left(a s_{\phi}+\right.\right.$ $\left.z) k_{1} \lambda_{2}+\left(a s_{\phi}-z\right) \lambda_{1} k_{2}\right], \quad q_{O 3}=p_{W 2} \mu_{3}$, $r_{O 3}=a s_{\vartheta} s_{\phi}\left[2 s_{\phi} k_{1} k_{2}+k_{1} \lambda_{2}\left(c_{\phi}+c_{\vartheta} s_{\phi}\right)+\right.$ $\left.\lambda_{1} k_{2}\left(c_{\phi}-c_{\vartheta} s_{\phi}\right)\right]$, and $\mu_{3}=2 c_{\phi} \lambda_{1} \lambda_{2}+$ $c_{\phi} c_{\vartheta}\left(k_{1} \lambda_{2}-\lambda_{1} k_{2}\right)+s_{\phi}\left(k_{1} \lambda_{2}+\lambda_{1} k_{2}\right)$.

\section{Singularity of PRoM-120}

\section{a) Constraint singularity}

The constraint singularity occurred if constraint wrench screws in the manipulator became linearly dependent [9]. For PRoM-120, it can be checked from each screw in the PWS, eq. (15), whether linearly dependent or not. The preliminary knowledge was the condition of two constraint forces, $\left(\widehat{\$}_{W 2}, \widehat{\$}_{W 3}\right)$, that perpendicular to each other. These two wrenches were linearly independent. Then, the thorough examination evinced that each screw in the
PWS was always linearly independent to each other. It meant that rathe nk of the PWS was always three. Therefore, PRoM-120 did not have constraint singularity.

\section{b) Inverse kinematic and input transmission singularity}

The inverse kinematic singularity (IKS) of PRoM-120 occurs if and only if

$\left|\mathbf{J}_{q}\right|=\lambda_{1} \lambda_{2} \lambda_{3}=0$,

for the non-deficient rank of $\mathbf{J}_{x}$. Eq. (21) can be fulfilled if one of $\lambda_{1}, \lambda_{2}$, or $\lambda_{3}$ was equal to zero. At the IKS, the manipulator will lose one or more DOF which enables it to resist forces and moments while the actuators are locked.

The input transmission singularity exists if any certain motion imposed by the $i^{\text {th }}$ actuator cannot be transmitted by the TWS of that limb [10]. It was defined as

$\widehat{\$}_{I i} \circ \widehat{\$}_{T i}=\lambda_{i}=0$,

for $i=1 ; 2$; 3. Reciprocal product of $\widehat{\$}_{I i}$ and $\widehat{\Phi}_{T i}$ in eq. (22) expressed that the twist screw of $i^{\text {th }}$ lithe $\mathrm{mb}$ was perpendicular to the transmission wrench screw of the $t^{\text {th }}$ limb. In other hand, it signified that the link $B_{i} C_{i}$ was parallel to the $X Y$ plane. Therefore, the singular condition given by the IKS was akin to condition of the input transmission singularity, as revealed previously by [5].

The perpendicularity condition for each limb was satisfied by the following condition

$\left\{\begin{array}{l}L=\left|R-a\left(c_{\phi}-c_{\vartheta} s_{\phi}\right)\right| ; \text { for } \lambda_{1}=0 \\ L=\left|R-a\left(c_{\phi}+c_{\vartheta} s_{\phi}\right)\right| ; \text { for } \lambda_{2}=0 \\ L=\left|R-a\left(1+s_{\vartheta}\right)\right| ; \quad \text { for } \lambda_{3}=0\end{array}\right.$.

Referring to eq. (23) can be obtained a condition for a length of the link $B_{i} C_{i}(i=1 ; 2 ; 3)$ which was $L \geq R-a$. Figure 2 presented three configurations of PRoM-120 at this type of singularity, as expressed mathematically in eq. (23) for $L$ was slight greater than $R-a$.

Thus, PRoM-120 with selected numerical values of its kinematic constants as given by Table 1 and the selected travel distance of the prismatic joints did not experience the IKS and the input transmission singularity. 

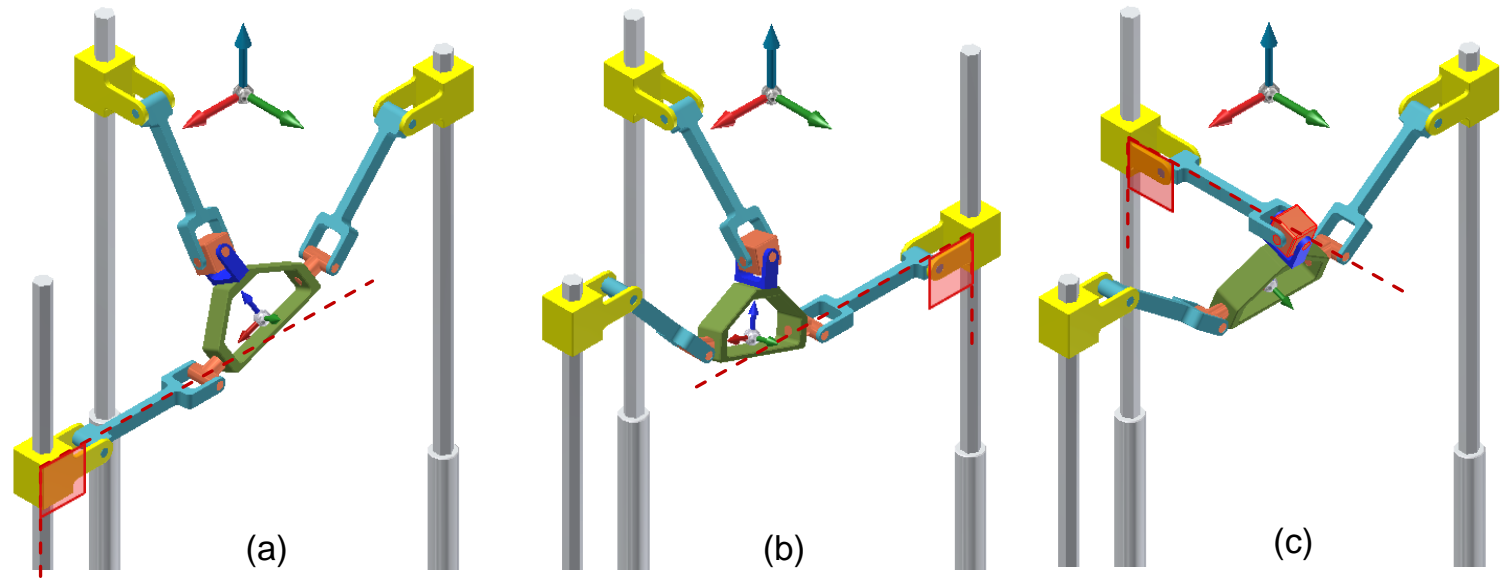

Figure 2. PRoM-120 configurations at the IKS or the input transmission singularity: (a) link $B_{1} C_{1}$, (b) link $B_{2} C_{2}$, and (c) link $B_{3} C_{3}$ were perpendicular to the first, second, and third prismatic joint, respectively.

\section{c) Direct kinematic and output transmission} singularity

The direct kinematic singularity (DKS) of PRoM-120 occurs if $\left|J_{x}\right|=0$ for the nondeficient rank of $\mathbf{J}_{q}$, or

$\left|\begin{array}{ccc}\lambda_{1} & h_{11} & h_{12}+a c_{\phi} \lambda_{1} \\ \lambda_{2} & h_{21} & h_{22}-a c_{\phi} \lambda_{2} \\ \lambda_{3} & h_{31}+a s_{\vartheta} c_{\phi} \lambda_{3} & a c_{\vartheta} s_{\phi} \lambda_{3}\end{array}\right|=0$.

In the singular condition, one or more DOF can be gained by the manipulator which enables it to perform infinitesimal motion even though no motion provided by the actuators. It also means that forces or moments cannot be held by the manipulator at this condition. Referring to eq. (24), it was hard to find the possibilities of the DKS. Alternatively, one can found from each column of $\left|\mathbf{J}_{x}\right|$, in which every element of each column must be zero, i.e. $\lambda_{1}=0 ; \lambda_{2}=0 ; \lambda_{3}=0$, or $h_{11}=0 ; h_{21}=0 ; h_{31}+a s_{\vartheta} c_{\phi} \lambda_{3}=0$, or $h_{12}+a c_{\phi} \lambda_{1}=0 ; h_{22}-a c_{\phi} \lambda_{2}=0 ; a c_{\vartheta} s_{\phi} \lambda_{3}=0$.

Then, the output transmission singularity occurs if any certain transmission wrench of the $i^{\text {th }}$ limb $\hat{\$}_{T i}$ cannot transmit power from the actuator of the $i^{\text {th }}$ limb to provide motion of the platform which represented by $\widehat{\$}_{O i}[10]$. It was defined as

$\widehat{\$}_{T i} \circ \widehat{\$}_{O i}=0$,

for $i=1 ; 2 ; 3$. The physical meaning behind this singular condition for each limb was a condition when the link $B_{i} C_{i}$ was in the plane to the platform plane. The platform plane was a plane formed by the point $C_{1}, C_{2}$, and $C_{3}$. In other words, the link $B_{i} C_{i}(i=1 ; 2 ; 3)$ was either in fully extended or fully folded condition with the platform. In reality, the last cannot be reached because of joint limitation and rigid body interferences among certain components.

When the link $B_{i} C_{i}(i=1 ; 2 ; 3)$ lying on the same plane with the platform plane, PRoM-120 could undergo some infinitesimal motion while the actuators were locked. Hence, it meant that the output transmission singularity was similar to the DKS which emerged previously by [5]. Figure 3 presented only three configurations of PRoM-120 when experiencing this type of singularity for $L$ was slight greater than $R-a$.

PRoM-120 with selected numerical values of its kinematic constants, as listed in Table 1, and the selected travel distance of the prismatic joints gave one configuration for this type of singularity. This configuration was the link $B_{3} C_{3}$ lying on the same plane with the platform plane.

\section{d) The combined singularity of the kinematic and the transmission singularity}

The combined kinematic singularity (CKS) arise when the inverse and the direct kinematic singularity occur concurrently. Also, it is the same with the combined transmission singularity, where both input and output transmission singularity coexisted. It has been shown by [5] for Tex3 parallel manipulator. 


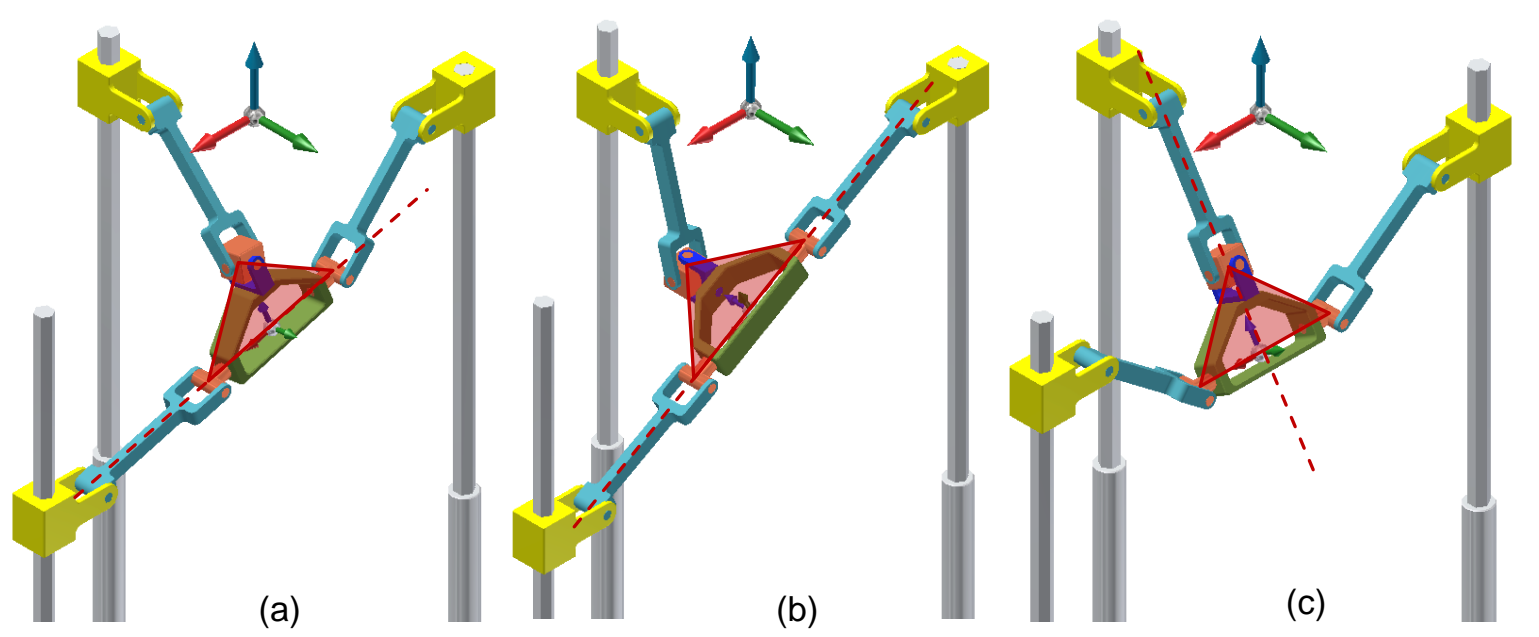

Figure 3. PRoM-120 configurations at the DKS or the output transmission singularity:

(a) link $B_{1} C_{1}$, (b) both link $B_{1} C_{1}$ and $B_{2} C_{2}$, and (c) link $B_{3} C_{3}$ were in the plane to the platform plane, respectively.
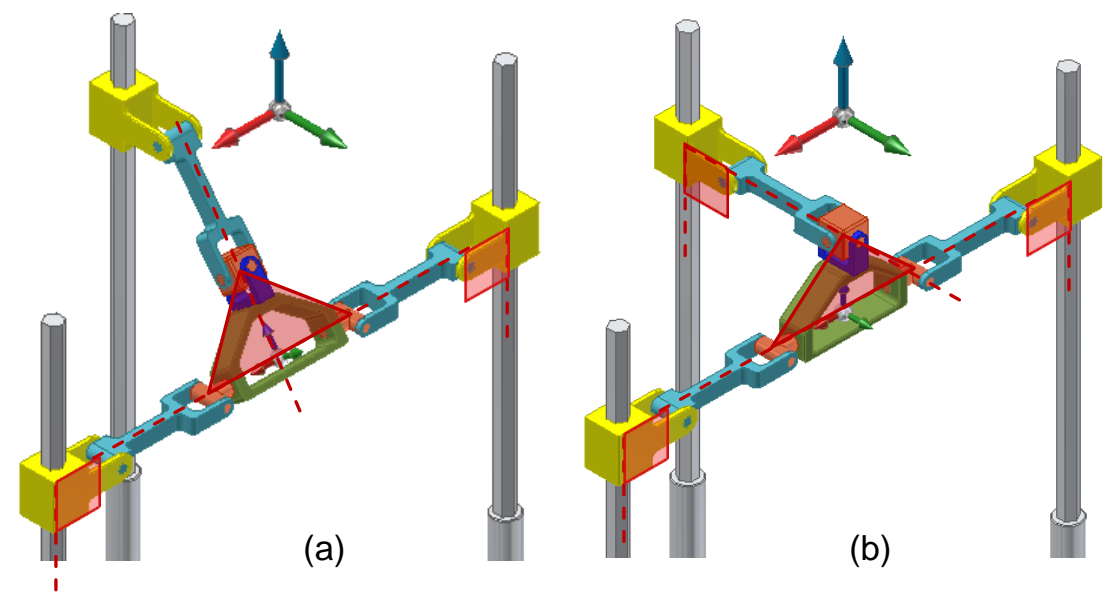

Figure 4. Two PRoM-120 configurations at the CKS or the combined transmission singularity: the first two links perpendicular to the first two prismatic joints and parallel to the platform plane while (a) the third links parallel to the platform plane and (b) the third limb perpendicular to the third prismatic joint.

This type of singularity happened for special architectural dimension, for PRoM-120, it was $L=R-a$. Figure 4 depicted two configurations of the these combined singularities for the condition of $L=R-a$.

The combined singularity did not occur 1 . for PRoM-120 with selected numerical values of its kinematic constants as listed previously in Table 1 and the selected travel distance of the prismatic joints. It was due to the fact that 2 . PRoM-120 did not possess the IKS and the input transmission singularity for the given numerical values of its kinematic constants.

\section{CONCLUSIONS}

Kinematic and singularity analysis of PRoM-120 had been presented in this paper. There were five items that could be outlined as the conclusions, that were

1. Kinematic analysis of PRoM-120, i.e. the inverse kinematic problem and the velocity equation, was a simple equation in closed form solution.

2. PRoM-120 did not possess constraint singularity because wrench system (the PWS) was always linearly independent or having rank equal to the number of wrench system, which was 3 . 
3. It was evinced and proved that the kinematic singularity had a close relationship with the transmission singularity. The IKS, the DKS, and the CKS were similar to the input, the output, and combined transmission singularity, respectively.

4. The IKS of PRoM-120 existed when one of the links perpendicular to each prismatic joint. Then, the DKS of PRoM120 occurred when one of the links was in the plane with the platform plane. Finally, the CKS happened if both the IKS and DKS occurred at the same time.

5. It was shown that only one singular condition known for PRoM-120 with the selected numerical values as given in Table 1, i.e. the third link was in the parallel to the platform plane. This singular condition fell to the DKS or the output transmission singularity.

Design and optimization of the kinematic constants had by PRoM-120 will be discussed in the future study.

\section{ACKNOWLEDGMENT}

The authors would like to express our gratitude to the General Directorate of Research and Development - The Ministry of Research, Technology and Higher Education that funded this research under the scheme "Penelitian Dosen Pemula" contract no. 052/K10/KM/KONTRAK-PENELITIAN/2018.

Also, the reviewers whom blind reviewed and give correction to this manuscript is gratefully acknowledged.

\section{REFERENCES}

[1] Z. Pandilov and V. Dukovski, "Comparison of the Characteristics Between Serial and Parallel Robots," Fascicule, vol. 1, no. 1, pp. 2067-3809, 2014.

[2] K. H. Hunt, "Structural kinematics of in and parallel and actuated robot arms," ASME J. Mech. Transm. Autom. Des., vol. 105, pp. 705-712, 1983.

[3] J. A. Carreterro, M. Nahon, C. M. Gosselin, and B. Buckham, "Kinematic analysis of a three-dof parallel mechanism for telescope application," in Proceeding of ASME Design Automation Conference, 1999, pp. 17-24.

[4] Q. Li, Z. Chen, Q. Chen, C. Wu, and X. $\mathrm{Hu}$, "Parasitic motion comparison of 3PRS parallel mechanism with different limb arrangements," Robot. Comput. Integr. Manuf., vol. 27, no. 2, pp. 389396, 2011.

[5] L. Xu, Q. Li, J. Tong, and Q. Chen, "Tex3: An 2R1T Parallel Manipulator with Minimum DOF of Joints and Fixed Linear Actuators," Int. J. Precis. Eng. Manuf., vol. 19, no. 2, pp. 227-238, 2018.

[6] S. Herrero, C. Pinto, O. Altuzarra, and C. Roldan-Paraponiaris, "Analysis and Design of the 2PRU-1PRS Manipulator for Vibration Testing," in Proceedings of the ASME 2014 International Mechanical Engineering Congress and Exposition, 2014, pp. 1-8.

[7] S. Herrero, C. Pinto, O. Altuzarra, and M. Diez, "Analysis of the 2PRU-1PRS 3DOF parallel manipulator: kinematics, singularities, and dynamics," Robot. Comput. Integr. Manuf., vol. 51, pp. 6372, 2018.

[8] C. M. Gosselin and J. Angeles, "Singularity Analysis of Closed-Loop Kinematic Chains," IEEE Trans. Robot. Autom., vol. 6, no. 3, pp. 281-290, 1990.

[9] D. Zlatanov, I. A. Bonev, and C. M. Gosselin, "Constraint singularities of parallel mechanisms," in Proceedings IEEE International Conference on Robotics and Automation, 2002, vol. 1, pp. 496-502.

[10] X.-J. Liu, C. Wu, and J. Wang, "A New Approach for Singularity Analysis and Closeness Measurement to Singularities of Parallel Manipulators," J. Mech. Robot., vol. 4, no. 4, p. 041001/1-10, 2012.

[11] J. Gallardo-Alvarado, Kinematic Analysis of Parallel Manipulators by Algebraic Screw Theory. Springer International Publishing, 2016. 